\title{
High-Reynolds-number weakly stratified flow past an obstacle
}

\author{
By S. I. CHERNYSHENKO ${ }^{1}$ AND IAN P. CASTRO ${ }^{2}$ \\ ${ }^{1}$ Institute of Mechanics, Moscow University, 117192 Moscow, Russia \\ ${ }^{2}$ Department of Mechanical Engineering, University of Surrey, Guildford, GU2 5XH, UK
}

(Received 9 February 1995 and in revised form 29 November 1995)

\begin{abstract}
Stably stratified steady flow past a bluff body in a channel is considered for cases in which the stratification is not sufficiently strong to give solutions containing wave motions. The physical mechanisms by which stratification influences the flow are revealed. In particular, the drag reduction under weak stratification, observed in experiments, is explained. This is achieved by constructing an asymptotic laminar solution for high Reynolds number $(R e)$ and large channel width, which explicitly gives the mechanisms, and using comparisons with numerical results for medium $R e$ and experiments for turbulent flows to argue that these mechanisms are expected to be common in all cases. The results demonstrate the possibility, subject to certain restrictions, of using steady high- $R e$ theory as a tool for studying qualitative features of real flows.
\end{abstract}

\section{Introduction}

The general problem of the viscous flow of a density-stratified fluid past an obstacle of arbitrary shape will, like the corresponding homogeneous flow, almost certainly never yield to a complete theoretical description. There have been many attempts to develop theories for simpler inviscid cases and some of these have been successful in revealing the dominant features of such flows, many of which have been confirmed by laboratory experiments. Practically all the extant literature, however, has been concerned with cases in which the density stratification leads to wave motions of various kinds, notably the lee waves that can be generated by topographic forcing of the flow. The well-known texts of Turner (1973) and Yih (1980) discuss typical ranges of problems in this general class that have been addressed. There have also been a number of more recent studies of the interaction between wave motions and a turbulent near wake - see Chomaz, Bonneton \& Hopfinger (1993) and Boyer et al. (1989) for examples of experimental studies of axisymmetric and planar cases in which the neutral flow wake was unsteady.

Much less attention has been paid to cases when the stratification is weak - in the sense that any internal wave motions generated have propagation speeds below the upstream flow velocity and so are swept downstream. In this sense the flow is supercritical. There is experimental evidence that for high-Reynolds-number flow over bodies which generate a separated wake region, stratification first acts (in the supercritical regime) to reduce both the size of this region and the obstacle drag (e.g. Davis 1969; Lofquist \& Purtell 1984; Castro, Snyder \& Baines, 1990). Castro et al. (and Hanazaki 1988) demonstrated via numerical computation that these effects also occur in low-Reynolds-number (laminar) flows although, again, interest was concentrated on the subcritical parameter regime in which wave motions interact with 
the near wake and may also propagate upstream. It was argued that in high-Re laboratory experiments the drag reduction could be explained on the basis of the inhibiting effects of stratification on vertical motions in the turbulent wake and on the pressure field changes arising from the tendency for constant-density surfaces to keep to their upstream height. This latter is an inviscid mechanism and it was not clear to what extent the stratification effects depended on it, rather than on the former. In turbulent flows stratification (of any strength) will also act to damp the vertical fluctuations and hence change the turbulence structure. Such effects have received considerable attention (e.g. Lin \& Pao 1979), but constitute a rather different problem than the one addressed here.

The purpose of this paper is to study the dominant physical mechanisms which cause stable density stratification to influence separated flow past a bluff body. We use a unified approach, combining the analysis of experimental, numerical and theoretical results. Experimental results are, naturally, the most realistic but have (in this case) the disadvantage of being the most difficult to use to identify physical mechanisms. On the other hand, the theoretical result - an asymptotic solution for steady flow at high $R e$ - clearly reveals the mechanisms but refers to a hypothetical laminar flow and cannot therefore be used in practice. The general idea for overcoming this difficulty, exploited in this paper, is that we compare experimental (high-Re, turbulent), theoretical (high$R e$, laminar and steady) and numerical (medium-Re, laminar, steady) results and observe that in spite of the differences in the parameter ranges and methods used, these results are similarly affected by stratification, both qualitatively and to the same order of magnitude. From this we argue that the mechanisms governing the influence of the stratification are common in all cases. The asymptotic investigation, therefore, does allow one to identify and understand physical mechanisms which are relevant to real flows and, in particular, sheds some light on the question raised above concerning the importance of inviscid mechanisms.

It must be recognized, however, that application of the asymptotic theory in order to reveal physical mechanisms of real turbulent flows is subject to certain restrictions. In this context the essential differences between high-Re laminar and real turbulent flows are twofold. First, the structure of an effective turbulent viscosity differs from that of the molecular viscosity - the latter is a scalar having a constant value throughout the laminar flow, whereas the former is a tensor with significant spatial variations. Second, in asymptotic theory the Reynolds number is assumed to tend to infinity while in a turbulent separated flow the effective Reynolds number (i.e. that based on the turbulent viscosity) is usually about 100 , which is not large enough for the asymptotic theory to give good quantitative agreement even with an exact laminar solution. Many features of the asymptotic flow cannot, therefore, be expected to be observed in real turbulent flows. This is especially true for those features that involve the specific differences mentioned above. The most obvious example is the influence of the Reynolds number on the flow.

Nonetheless, high-Reynolds-number steady laminar flows over bluff bodies have much in common with the corresponding (averaged) turbulent flows. In both cases the flow near the body has a Kirchhoff structure, there is a recirculating eddy flow in the near wake, the size of this eddy can be large compared with the body size, and so on. If some additional physical effect introduced into the flow manifests itself mainly through the common features, rather than those that are different, then the physical mechanisms of this effect on the flow can be expected to be the same in the asymptotic theory as in the real flow. Density stratification does manifest itself in this way, as we will show, and it is on that basis that we believe that our attempt to reveal the physical 
mechanisms of a real flow using asymptotic theory is reasonable. Note that if the physical mechanisms were not of such a nature that they could be expressed in a form independent of the asymptotic considerations, the above argument would be inadmissible.

To achieve our purpose, all three approaches - laboratory experiment, numerical experiment and analytical theory--are equally important. However, since both laboratory experiments and (to some degree) numerical calculations have been described earlier, a considerable part of this paper is concerned with the theoretical study. This is an extension of the theory for neutral flow past a bluff body (Chernyshenko 1988, referred to herein as CH1) or its variant for the flow through a cascade of bluff bodies (Chernyshenko \& Castro 1993, referred to herein as CH2). To comprehend the work completely the reader should be familiar with at least one of these papers; the latter is the more complete. However, the present paper is selfcontained in the sense that, with acceptance of the validity of the purely technical statements concerning the construction of the asymptotic expansions, the physical nature of our results can be understood without consulting previous work.

The problem under consideration is that of a steady plane flow of stably stratified fluid past a bluff body in a channel. The body possesses a symmetry plane coinciding with that of the channel and for simplicity a zero tangential stress condition is imposed at both channel walls. Hence the upper wall may be considered as a free surface whose shape is not essentially deformed by the fluid motion. At the lower wall this condition implies that there is no boundary layer. Unlike those used in the low-Reynolds-number numerical experiments of Castro (1993), these boundary conditions do not quite correspond to an experiment in a towing tank, in which the body is towed along at the centre of the water depth. In fact, most such experiments-particularly those undertaken to simulate topographical flows - use bodies towed along the free surface (or along the bottom of the tank) which would then correspond to the imposed symmetry axis in the present case. In such cases the other boundary has a velocity equal to that in the upstream flow, i.e. zero, rather than a zero-stress condition. The density far upstream is taken to decrease linearly with the vertical coordinate, with a variation small enough to allow the Boussinesq approximation to be applied, and it is assumed for convenience that this variation is achieved via a solute (salt, in a physical experiment) rather than via temperature. The theory could equally well be developed in the latter terms (but see $\$ 2$ ).

With these assumptions the flow is governed by the following equations:

$$
\begin{gathered}
\boldsymbol{u} \cdot \boldsymbol{\nabla u}=-\nabla p+(1 / \operatorname{Re}) \nabla^{2} \boldsymbol{u}-(\operatorname{Ri} \rho / H) \boldsymbol{j} \\
\boldsymbol{u} \cdot \boldsymbol{\nabla} \rho=(1 /(\operatorname{Re} S c)) \nabla^{2} \rho, \\
\boldsymbol{\nabla} \cdot \boldsymbol{u}=0 .
\end{gathered}
$$

Here $u$ is the velocity vector normalized by the velocity far upstream, $U_{\infty}^{*}$, which is assumed uniform, $p$ is the pressure normalized by $\rho U_{\infty}^{* 2}, R e=U_{\infty}^{*} R^{*} / \nu, R^{*}$ is the characteristic size of the body, $\nu$ is the kinematic viscosity, $R i$ is the Richardson number defined by $-\left[\left(g H^{* 2} / \rho^{*} U_{\infty}^{* 2}\right) \partial \rho^{*} / \partial y\right]$ (with the density gradient here referred to conditions far upstream), $H^{*}$ is the channel half-width and $H=H^{*} / R^{*}$. Stars denote dimensional values. In (1.1) $\rho$ is the density variation normalized by the upstream quantity $R^{*} \partial \rho^{*} / \partial y$ and $S c$ is the Schmidt number (i.e. the ratio of the coefficient of diffusion to the kinematic viscosity). For brevity $\rho$ is called density in what follows. Because of symmetry it is sufficient to consider only the upper part of the flow field. The 
'weak' stratification case considered here is defined by $R i<\pi^{2}$, because for larger values the flow is subcritical for at least one wave mode. The boundary conditions are

$$
\begin{gathered}
x \rightarrow-\infty, \quad u \rightarrow 1, \quad \rho \rightarrow-y, \quad \partial u / \partial y=0, \\
y=0, \quad \partial u / \partial y=0, \quad \rho=0 \quad \text { outside the body, } \\
y=H, \quad \partial u / \partial y=0, \quad \rho=-H,
\end{gathered}
$$

and, on the body surface,

$$
\boldsymbol{u}=0, \quad \partial \rho / \partial n=0 .
$$

Here, (1.5) follows from symmetry, (1.6) is the impermeability and zero-stress condition on the free surface and a somewhat artificial condition for $\rho$ which makes the upstream condition (1.4) consistent. As we will tend $S c$ to infinity (see below) this condition is not in fact important.

\section{First limit: $S c \rightarrow \infty$}

The problem under consideration contains four parameters: $R e, R i, S c$ and $H$. To simplify, let $S c$ tend to infinity with constant $R e, R i$ and $H$. Equation (1.2) then tends to $\boldsymbol{u} \cdot \boldsymbol{\nabla} \rho=0$, whose general solution is $\rho=\rho(\psi)$ where $\psi$ is the stream function. The boundary condition (1.4) yields $\rho(\psi)=-\psi$ for $\psi>0$. For $\psi<0$ (inside the separated eddy behind the body) a theorem similar to the well-known Prandtl-Batchelor theorem can easily be proved (see Kamachi, Saitou \& Honji 1985). That is, in the limit $S c \rightarrow \infty$ inside the region of closed streamlines, $\rho=$ const. The value of this constant could be found by asymptotic methods in approximately the same way as the value of the vorticity inside the eddy is found below for $R e \rightarrow \infty$, but the analysis would be lengthy. It would also, in fact, be unnecessary since it can be shown much more directly that in the present case this constant equals zero, as follows.

Consider a region inside the contour ABCDEFGA (figure 1), with BC being a streamline. For finite $S c$ the density $\rho$ satisfies equation (1.2) and therefore (Courant 1962) the maximum principle holds: i.e. the density has a maximum and a minimum on the contour ABCDEFGA. Moreover, at the maximum $\partial \rho / \partial n \neq 0$ (see Courant 1962, lemma in item 4, section 6 of chapter 4). Hence the density cannot have an extremum at the body surface on which $\partial \rho / \partial n=0$. The symmetry line must also be excluded since $\rho=0$ there. Therefore $\left|\rho_{\text {eddy }}\right| \leqslant \max |\rho|(\mathrm{ABCD})$. Moving the left and right boundaries ( $\mathrm{AB}$ and $\mathrm{CD}$ ) to infinity we obtain $\left|\rho_{\text {eddy }}\right| \leqslant \max |\rho|\left(\psi=\psi_{0}=\right.$ const). This means the density inside the eddy is not greater than the maximum density on the streamlines outside. This is valid for any $S c$. For $S c \rightarrow \infty$ outside the eddy $\rho=\rho\left(\psi_{0}\right)=-\psi_{0}$ on the eddy and body scale, as explained above. This equality also holds far downstream in the wake because $\rho \rightarrow-y$ and $u \rightarrow 1$ for $x \rightarrow \infty$. Therefore, choosing sufficiently large $S c$ and sufficiently small $\left|\psi_{0}\right| \neq 0$ it is easy to prove that the limit of $\left|\rho_{e d d y}\right|$ as $S c \rightarrow \infty$ is not greater than any given $\left|\psi_{0}\right|>0$ and hence that this limit must be zero. The subsequent analysis will be limited to the case $S c=\infty$, so that the problem under consideration is now that of equations (1.1), (1.3)-(1.7) with the density determined by

instead of (1.2).

$$
\left.\begin{array}{ll}
\rho=\rho(\psi)=-\psi, & \psi>0, \\
\rho=\rho(\psi)=0, & \psi \leqslant 0,
\end{array}\right\}
$$

Note that this case $(S c \rightarrow \infty)$ is particularly relevant to experiments in which the density stratification is achieved using salt water, for which $S c=O(1000)$. It is less appropriate for cases in which the density is essentially related directly to temperature 


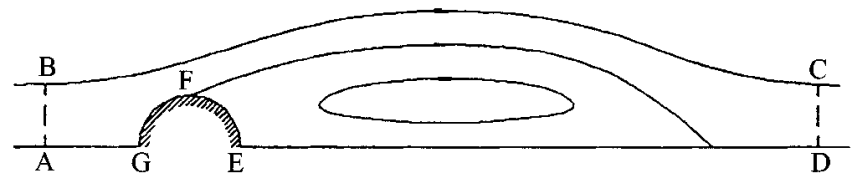

FIGURE 1. Sketch of the flow.

(i.e. in thermally stratified flows) since then the Prandtl number, which has the same dynamical significance as $S c$, is usually $O(1)$. In that case the density distribution cannot be found explicitly as a function of $\psi$. However, when $R e \rightarrow \infty$ a theorem similar to the Prandt1-Batchelor theorem again holds true: inside the region of closed streamlines $\rho=$ const. Moreover, in this region $\rho=0$ again, for the same reasons as given earlier, and outside the region $\rho=-\psi$. As the gradient of $\rho$ is not continuous, this density distribution is not valid in the vicinity of the discontinuity, i.e. near the eddy boundary. There is therefore a diffusion boundary layer in which the density differs from that given by (2.1). In the case $R e=O(1), S c \rightarrow \infty$ the thickness of this layer tends to zero as $1 / S c^{1 / 2}$, so that (2.1) becomes valid in the limit everywhere. In the case $R e \rightarrow \infty, S c=O(1)$ the thickness of the diffusion layer has the same order as the thickness of the vorticity layer (see below). Hence in this case (2.1) is valid only outside the vorticity boundary layer, while inside it the density distribution should be found as a solution to the corresponding equations. Now, the overall characteristics depend on the density distribution in the layer surrounding the eddy only via the value of $g_{\infty}$ (see below). Therefore, those of our results which do not depend strongly on the particular value of $g_{\infty}$ also hold true in the case $S c=O(1)$.

\section{The asymptotics for $H \sim R e, R i=0, R e \rightarrow \infty$}

The objective of this work is to provide insight into the complicating influence of stratification on the separated region. Previous results on the high-Reynolds-number asymptotics of separated flows $(\mathrm{CH} 1$ and $\mathrm{CH} 2)$ provide a convenient tool for this. We first review briefly the main features of the theory given in $\mathrm{CH} 2$. The problem considered there was that defined by equations $(1.1),(1.3)-(1.7)$ for $R i=0$. The ratio $H / R e$ was assumed to remain constant as $R e \rightarrow \infty$. It was shown that in this case both the eddy length and width have order $R e$. The asymptotic limit $R e \rightarrow \infty$ is not a uniform one: the nature of the limiting flow depends on the behaviour of the coordinates $x$ and $y$ as $R e \rightarrow \infty$. If, for example, $x, y=O(1)$, then the reattachment point moves to downstream infinity with $R e$ tending to infinity. Hence the eddy becomes open. The channel wall also moves upward to infinity and the flow on the $x, y=O(1)$ scale was shown to be the Kirchhoff free-streamline flow. Although on $x, y=O(1)$ scales the eddy extends to infinity downstream, it does not on larger scales. For this reason the velocity on the free streamline is not unity but is determined by matching the flow on the larger scales. In the Kirchhoff flow the drag is related to the velocity on the free streamline by the formula

$$
c_{d}=k_{d} V^{2},
$$

where $c_{d}$ is the drag coefficient, $k_{d}$ is the Kirchhoff drag coefficient with a freestreamline velocity equal to unity and $V$ is the actual free-streamline velocity. This relationship will be used later. We also note for future use that in the Kirchhoff flow vorticity is constantly being shed from the body and moves downstream in the mixing layer along the free streamline. The vorticity flux along the (upper) streamline is

$$
F=-V^{2} / 2 \text {. }
$$


The limiting flow looks quite different in another distinguished limit. Let $x, y=$ $O(R e)$ for $R e \rightarrow \infty$. Then on this scale the body shrinks to a point and vanishes in the limit. In contrast, as $H=O(R e)$, the channel walls and the whole eddy remain present on this scale. As the length scale is large, the effective Reynolds number is also large. Hence the limiting flow on this scale is inviscid. Now, the only suitable choice for the limiting flow is the Sadovskii flow in a channel, considered by Chernyshenko (1993, referred to herein as $\mathrm{CH} 3$ ). This flow contains two closed-streamline regions touching along the centreline of the channel. Inside these streamlines the vorticity is constant by the Prandtl-Batchelor theorem. Outside, the flow is potential. It is proved in $\mathrm{CH} 2$ that in the limiting flow the drop in the Bernoulli constant on the eddy boundary equals zero. This flow depends only on the ratio, $H / L$, of the channel half-width to the eddy length. It was also proved that to leading order the energy dissipation due to viscous forces can be calculated from the flow on this scale. Using the Bobylev-Forsythe theorem (Serrin 1959) the drag coefficient can be calculated as

$$
c_{d}=(1 / R e) \iint \omega^{2} \mathrm{~d} x \mathrm{~d} y,
$$

in which the integration is over the entire flow, i.e. on both sides of the channel centreline. Here $\omega$ is vorticity and $c_{d}=\mathrm{drag} /\left(\rho^{*} U_{\infty}^{* 2} R^{*}\right)$. As $R e \rightarrow \infty$ the integral tends to that over the eddy-scale Sadovskii flow alone. Note that if there is no wave drag the latter result for the drag is valid in the case of a stratified flow, although the vorticity distribution is different. Now, in Sadovskii flow

$$
\iint_{\substack{\text { eddy-scale } \\ \text { Sadovskii flow }}} \omega^{2} \mathrm{~d} x \mathrm{~d} y=C(H / L) .
$$

The reason is that in Sadovskii flow for a fixed upstream velocity $\omega$ is inversely proportional to $L$ :

$$
\omega=C_{1}(H / L) / L .
$$

Hence when $L$ changes, $C$ remains constant. We therefore arrive at

$$
c_{d}=C(H / L) / R e .
$$

The action of viscosity leads to diffusion of vorticity from the eddy and toward the centreline, where it equals zero, and this loss of vorticity in the eddy is balanced by the vorticity shed from the body. In the Sadovskii flow there is a discontinuity in vorticity at the eddy boundary and on the centreline inside the eddy so that a vorticity boundary layer is present. With this layer having a thickness of order unity the vorticity flux needed to balance the loss of vorticity from the eddy can be estimated as

$$
F=C_{2} \omega_{e d} L / R e .
$$

Here $\omega_{e d}$ is the vorticity inside the eddy in the Sadovskii flow. To calculate $C_{2}$ a careful examination of the boundary layer surrounding the eddy is needed. The important point to note here is that with $H / L$ given the Sadovskii flow can be calculated and then the boundary layer can also be calculated. Hence the five equations (3.1)-(3.5) enable the five unknowns $\omega_{e d}, L, c_{d}, V$ and $F$ to be found. There are several other distinguished limits apart from those mentioned thus far; these are considered in $\mathrm{CH} 1$ and $\mathrm{CH} 2$. In particular, it is shown that the $F$ in equations (3.2) and (3.5) are identical: that is, there is no vorticity flux loss in the various additional regions corresponding to distinguished limits between $x, y=O(1)$ and $x, y=O(\mathrm{Re})$. Further details can be found in $\mathrm{CH} 2$. 


\section{The asymptotics for $H \sim R e, R i \sim 1, R e \rightarrow \infty$}

Extension of the earlier theory to the stratified flow case is straightforward. For this reason, the technical questions regarding the asymptotics (such as choosing the characteristic limits, matching, etc.) are explained and discussed below only if this is necessary for the main objective. We assume by analogy with the previous theory (CH2) that $H$ tends to infinity with $R e$ in such a way that $H / R e$ remains constant and we will restrict ourselves to the case without waves, so that $R i<\pi^{2}$.

For $R i \sim 1$ the influence of stratification is significant only in the distinguished limits having a length scale of order $H \sim R e$, because on smaller scales the term with the density variation vanishes from equation (1.1) in the limit $R e \rightarrow \infty$. For $R i=0$ there are only two distinguished limits of this scale - the eddy-scale flow and the recirculating boundary layer (see $\mathrm{CH} 2$ ). Naturally, stratification may change the asymptotic structure itself (i.e. the number and nature of the distinguished limits) but we may reasonably hope that such changes are not significant, at least for weak stratification. The self-consistency of the expansion constructed here supports this conclusion. Therefore, the regions on smaller scales may be assumed to have the same structure as for the $R i=0$ case. Properties of the flows on these scales needed for the analysis in this paper are pointed out below and other details of these flows can be found in $\mathrm{CH} 2$. We consider only the two regions directly affected by stratification.

\subsection{The eddy-scale flow}

The proper length scale for the eddy is $R e$. Recognizing that $H \sim R e$, it is convenient to introduce new independent variables $X=x / H, Y=y / H$. For $\operatorname{Re} \rightarrow \infty$ the expansion of the stream function $\psi$ on the eddy scale is $\psi(x, y, R e, R i, H)=H \Psi(X, Y, H / R e$, $R i)+\ldots$ In the limit $R e \rightarrow \infty, R i=$ const, $H / R e=$ const the viscous term vanishes from equation (1.1). Using equation (2.1) the resulting problem can then be formulated as follows:

$$
\begin{aligned}
\nabla^{2} \Psi & =-\operatorname{Ri}(\Psi-Y), & & \Psi>0 \\
& =\Lambda=\mathrm{const}, & & \Psi<0 .
\end{aligned}
$$

The Prandtl-Batchelor theorem (which shows that the vorticity, $A$, is constant inside the eddy) is valid in this case because inside the eddy there is no stratification, see $\S 2$. The boundary conditions are $Y=0, \Psi=0 ; Y=1, \Psi=1 ; X \rightarrow \pm \infty, \Psi \rightarrow Y$. For the given values of $R i$ and $L$ this problem has a one-parameter family of solutions depending on the ratio of eddy length to channel width (see $\mathrm{CH} 3$ ). Choice of the correct member of this family can only be made after matching with the other regions. Note that the vorticity distribution in this flow, expressed in terms of the original variables $(x, y$, etc $)$ is

$$
\begin{aligned}
\omega & =\operatorname{Ri}(\psi-y) / H^{2}, & & \psi>0 \\
& =-\Lambda / H, & & \psi<0 .
\end{aligned}
$$

The problem defined by equation (4.1) was solved numerically in $\mathrm{CH} 3$.

\subsection{The recirculating boundary layer}

The recirculating boundary layer may be associated with the leading term in the vorticity expansion or the second term in the velocity expansion. This follows from the relation $\omega=\operatorname{rot} u$ combined with the continuity of the velocity field in the Sadovskii flow. Since it is neater, we use the vorticity representation and introduce independent 


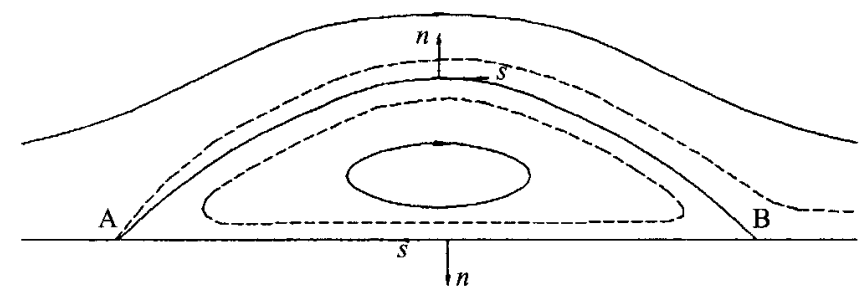

FIGURE 2. The recirculating region, sketched on the eddy scale (so that the body at A disappears). The dashed line is the boundary of the recirculating shear layer.

boundary layer variables as shown in figure 2, with the length scale for $s$ and $n$ the same as for $x$ and $y ; s=0$ is at the point A. Usually new scales and a new notation are introduced for every distinguished limit but for brevity we use the same scales and notation, with the implication that only the leading terms are retained. Accordingly, in the boundary layer, $s \sim R e$. Then to keep the viscous terms in the boundary layer of the same order as the convection terms it is necessary to assume $n \sim 1$. Hence the stream function in the boundary layer is also of order unity. The vorticity equation (corresponding to equation (1.1)) is

$$
u \cdot \nabla \omega=(1 / R e) \nabla^{2} \omega+\left(R i / H^{2}\right) v \partial \rho / \partial \psi .
$$

Here $v$ is the $y$-component of the velocity. Since the velocity field is continuous in the inviscid flow, the leading term for the velocity in the boundary layer equals the velocity in the inviscid flow and does not change across the layer. Denoting this leading term by $U(s)=|u|$ and, using a boundary layer approximation, we obtain the following equation in Mises variables:

$$
U \partial \omega / \partial \psi=\left(U^{2} / R e\right) \partial^{2} \omega / \partial \psi^{2}+R i U \sin \Theta \mathrm{d} \rho / \mathrm{d} \psi .
$$

Here $\Theta$ is the angle between the $x$-axis and the direction of the inviscid Sadovskii flow at the corresponding point of the eddy boundary. The order of the vorticity here is the same as in the eddy-scale flow. As the eddy size is of order $R e$ and the velocity of order unity, the vorticity is of order $1 / R e$. Hence all terms in equation (4.3) are of the same order. This equation is valid in the mixing layer between points $\mathrm{A}$ and $\mathrm{B}$ (see figure 2) and also along the centreline between $B$ and $A$. In the vicinity of these points new distinguished limits must be introduced. As noted earlier, these limits (and regions) do not differ greatly from those in neutral flow $(\mathrm{CH} 2)$ and will not be discussed in detail. It should be noted, however, that the mass flux through these regions is the same as in the boundary layer and is therefore of order unity. Hence the product of the length and velocity scales in these regions is of order unity also, so the effective Reynolds number calculated via these scales is of order $R e$. For this reason, the flow in the regions where the fluid from the boundary layer turns is effectively inviscid. Hence in these regions $\omega=\omega(\psi)$. This makes it possible to ignore these regions and to consider the centre-line boundary layer as a continuation of the mixing layer and vice versa. As a result, equation (4.3) is to be solved in the region shown in figure 3. Part of it $-0<s<s_{B}$, $-\infty<\psi<+\infty$-corresponds to the mixing layer and the rest $-s_{B}<s<s_{A}$, $-\infty<\psi<0-$ corresponds to the centreline boundary layer. At $s=s_{B}$ the vorticity is continuous for $\psi<0$ due to its conservation in the effectively inviscid region near point B. Analogously the vorticity is the same at $s=0$ and $s=s_{A}$ for $\psi<0$. This yields the condition

$$
\psi<0, \quad \omega(0, \psi)=\omega\left(s_{A}, \psi\right) .
$$




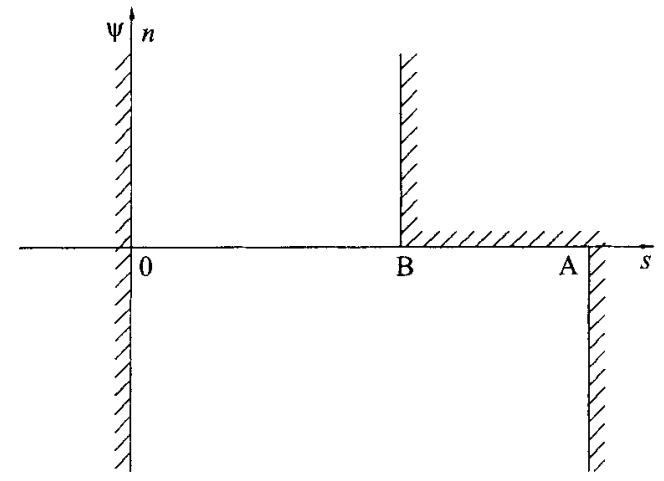

FIGURE 3. The region in which solutions to equation (4.3) are sought.

To establish an initial condition for $s=0, \psi>0$ we must consider the inviscid nature of the flow in the turn region near point $\mathrm{A}$. The vorticity distribution inside this region is determined by the inviscid formula (4.2). At $s=0, \psi=0$, this yields $\omega=$ $\operatorname{Ri}(\psi-y) / H^{2} \ll 1 / R e$, because $H \sim R e$ and in the turn region $\psi \sim 1$ and $y \ll R e$. Hence this initial vorticity is negligible in comparison with the vorticity scale in the boundary layer, which is $1 / R e$. The initial condition is therefore

$$
s=0, \quad \psi>0, \quad \omega(0, \psi)=0 .
$$

There is also the evident symmetry condition

$$
\psi=0, \quad s_{B}<s<s_{A}, \quad \omega=0 .
$$

The body is constantly shedding vorticity, which enters the boundary layer as a singular source at $s=0, \psi=0$. This yields the condition

$$
s=0, \quad \psi=0, \quad \omega=F \delta(\psi),
$$

where $F$ is the vorticity flux and $\delta(\psi)$ is a delta function.

Properties of boundary value problems of this kind have been widely studied (see Chernyshenko 1982, for a discussion and references). The solution of equations (4.3)-(4.7) is unique if the additional condition

$$
\psi \rightarrow-\infty, \quad|\omega|<\infty
$$

is applied. Hence the solution of (4.3)-(4.8) determines the limit $\lim _{\psi \rightarrow-\infty} \omega=\omega_{\infty}$. This $\omega_{\infty}$ must be equal to the value of the vorticity in the eddy $\omega_{e d}=-\Lambda / H$ (see (4.2)) in order to provide the matching of the boundary layer and eddy-scale flow.

Note now that the problem specified by equations (4.3)-(4.8) is linear. Hence $\omega_{\infty}$ can be represented as the sum of the value corresponding to non-stratified flow with the same $U(s), \Theta(s)$ and $R e$ and the value due to stratification. Moreover, the first of these is proportional to $F$ and the second to $R i / H^{2}$. Using the previous analysis of the nonstratified problem (Chernyshenko $1982 ; \mathrm{CH} 1 ; \mathrm{CH} 2$ ) it follows that

$$
\omega_{\infty}=2 D_{0}(b) F\left[2 \operatorname{Re} /\left(\omega_{e d} S\right)\right]^{1 / 2}+\omega_{s t \infty},
$$

in which the first term on the right-hand side is equation (2.13) in $\mathrm{CH} 2 . \omega_{e d}$ is the value of the vorticity inside the eddy and $S$ is the total area of both halves of the eddy so that by Stokes' theorem

$$
-\omega_{e d} S=2 \int_{0}^{s_{A}} U(s) \mathrm{d} s .
$$


In (4.9)

$$
b=\int_{0}^{s_{B}} U(s) \mathrm{d} S / \int_{0}^{s_{A}} U(s) \mathrm{d} s .
$$

The function $D_{0}(b)=D(0, b)$ in the notation of Chernyshenko (1982) and is tabulated there (see also CH2). Also, $\omega_{s t \infty}=\lim _{\psi \rightarrow-\infty} \omega_{s t}$, where $\omega_{s t}$ is the solution of (4.3)-(4.8) with $F=0$.

We now introduce the new independent variables

and denote

$$
t=\int_{0}^{s} U(s) \mathrm{d} s / \int_{0}^{s_{A}} U(s) \mathrm{d} s, \quad z=\psi\left(\operatorname{Re} / \int_{0}^{s_{A}} U(s) \mathrm{d} s\right)^{1 / 2}
$$

$$
\omega_{s t}=g(t, z) \frac{R i}{H^{2}} \int_{0}^{s_{A}} U(s) \mathrm{d} s .
$$

Then the problem (4.3)-(4.8) becomes (noting that $F=0$ for $\omega_{s t}$ )

$$
\left.\begin{array}{rl}
\partial g / \partial t=\partial^{2} g / \partial z^{2}+f(t), \quad f(t)= \begin{cases}-1, & z>0 \\
+0, & z<0\end{cases} \\
t=0, \quad z>0, \quad g=0, \\
z<0, \quad g(0, z)=g(1, z), \\
z \rightarrow-\infty, \quad|g|<\infty, \\
z=0, \quad b<t<1, \quad g=0, \\
f(t)=\sin \Theta / U .
\end{array}\right\}
$$

Denoting $\lim _{z \rightarrow-\infty} g=g_{\infty}$ and using (4.10) yields $\omega_{s t \infty}=-g_{\infty} \omega_{e d} S R i /\left(2 H^{2}\right)$. Denoting $C_{0}=\omega_{e d}{ }^{2} S$ and using the matching condition $\omega_{\infty}=\omega_{e d}$ the formula (4.9) can be rewritten as

$$
\frac{\operatorname{Re} F^{2}}{\omega_{e d}}=-\frac{C_{0}\left(1+g_{\infty} R i S / 2 H^{2}\right)}{8 D_{0}^{2}(b)} .
$$

The reason for introducing $g_{\infty}, C_{0}$ and $b$ is that they are uniquely determined if $\Psi(x, y)$, which is the solution of (4.1), is known. Hence for the particular member of the family of Sadovskii flows these quantities are determined. The method of calculating $g_{\infty}$ is given in the Appendix.

\subsection{The overall relations}

As discussed in $\S 3$ the drag can be calculated via the rate of energy dissipation. The same reasoning can be employed in the stratified case and gives the same formula as (3.4), except that $C$ now depends not only on $H / L$ but also on $R i$ :

$$
c_{d}=C(H / L, R i) / \text { Re. }
$$

Note that $C$, like $C_{0}$ and $g_{\infty}$ above, depends only on the particular choice of the member of the Sadovskii flow family, that is the choice of $A$ in (4.1) or $H / L$ (provided that the velocity scale is fixed equal to the velocity at infinity). If the size of the eddy

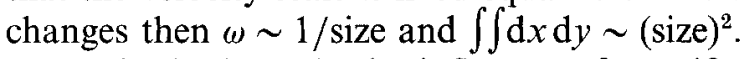

On the body scale the influence of stratification is characterized by the value of $R i / H^{2} \sim 1 / R e^{2}$ and is therefore negligible. The flow on the body scale is therefore the 
non-stratified Kirchhoff flow. This allows determination of the velocity on the free streamline. Hence equations (3.1), (3.2) and (4.14) give

$$
F=-C /\left(2 k_{d} R e\right) \text {. }
$$

Here $k_{d}$ is the usual Kirchhoff drag coefficient. Substitution of (4.15) in (4.13) gives

$$
\omega_{e d} k_{d}^{2} R e=-\frac{2 C^{2} D_{0}^{2}(b)}{C_{0}\left(1+g_{\infty} R i S / 2 H^{2}\right)^{2}} .
$$

Denoting $\alpha=S / L^{2}$ where $L$ is the eddy length we obtain

$$
\begin{aligned}
\frac{L}{k_{d}^{2} R e} & =\frac{1}{2} \frac{C_{0}^{3 / 2}\left(1+g_{\infty} R i S / 2 H^{2}\right)^{2}}{\alpha^{1 / 2} C^{2} D_{0}^{2}(b)}, \\
\frac{H}{k_{d}^{2} R e} & =\frac{1}{2} \frac{H C_{0}^{3 / 2}\left(1+g_{\infty} R i S / 2 H^{2}\right)^{2}}{L \alpha^{1 / 2} C^{2} D_{0}^{2}(b)} .
\end{aligned}
$$

For specified values of $H /\left(k_{d}^{2} R e\right)$ and $R i$ the parameter determining the Sadovskii flow ( $A$ in (4.1) or, alternatively, $H / L$ because $\Lambda=\Lambda(H / L, R i)$ ) can be found from (4.18). All other flow characteristics are then given by the previous formulae. In fact, we use equations (4.14)-(4.18) as a parametric representation for all quantities as functions of $H /\left(k_{d}^{2} R e\right)$ and $R i$. The quantitative results given below are based on the Sadovskii flow calculation described in $\mathrm{CH} 3$, although more data than were given there are used here.

\section{Results and discussion}

\subsection{The asymptotic results}

This theory is constructed for $R e \rightarrow \infty, H / R e=$ const, but it remains valid for all $R e \gg 1, H \gg 1$. The explanation is given in $\mathrm{CH} 2$ and is not repeated here. In $\mathrm{CH} 2$ there is an extensive discussion of the accuracy of the asymptotic results for given values of $H$ and $R e$. It was argued that this accuracy depends essentially on the value of the velocity on the free streamline on the body scale. Combining equations (3.2) and (4.15) gives for this velocity the expression:

$$
V^{2} k_{d} R e=C .
$$

Multiplying (4.18) by $V^{2} k_{d} R e$ yields

$$
\frac{V^{2} H}{k_{d}}=\frac{1}{2} \frac{H C_{0}^{3 / 2}\left(1+g_{\infty} R i S / 2 H^{2}\right)^{2}}{L \alpha^{1 / 2} C D_{0}^{2}(b)} .
$$

The right-hand sides of these two equalities depend only on $R i$ and $H / L$. Fixing a particular value of $V^{2}=\epsilon$, say, and varying $H / L$ for fixed $R i$, we obtain in the plane $\epsilon k_{d} R e, \epsilon H / k_{d}$ the curve at which $V^{2}=\epsilon$. Figure 4 shows such curves for various values of $R i$. Above each curve $V^{2}<\epsilon$. As discussed in $\mathrm{CH} 2$, the smaller the value of $V^{2}$ the greater the accuracy of the asymptotic results. It is clearly seen that with $R i$ increasing the region in which $V^{2}<\epsilon$ expands, so for increasing $R i$ the accuracy of the asymptotic results is expected to improve. To put it another way, the Reynolds number at which the asymptotic results may be representative of the flow should be significantly lower in the stratified case than in the corresponding neutral case. Further discussion of this and related questions (see $\mathrm{CH} 2$ ) lies beyond the scope of the present paper. Note, however, that the numerical calculations described by Castro (1993) and used here in comparisons were for $R e=50, R i=0-\pi^{2}$ and $H$ in the range 5-50, so are strictly 


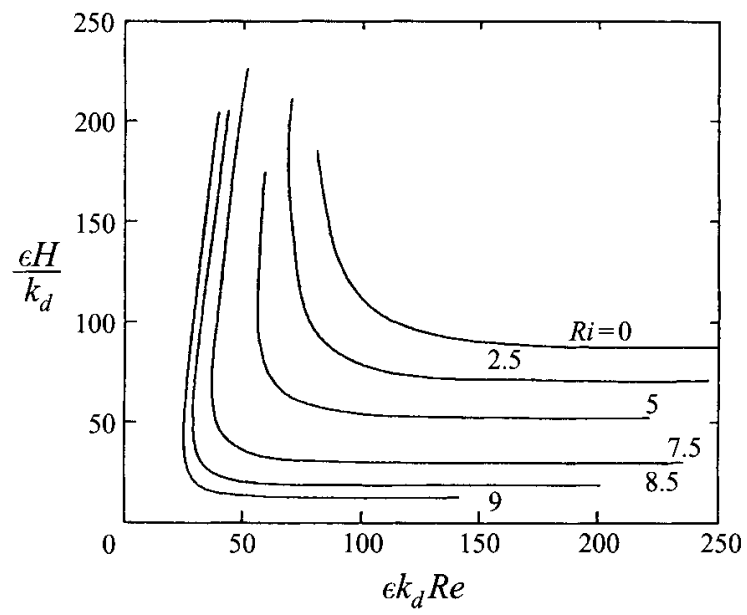

FiguRE 4. Curves of $V^{2}=\epsilon$ for various Ri. At a specific Ri the asymptotic solutions are best in the region above the corresponding curve, for which $V^{2}<\epsilon$.

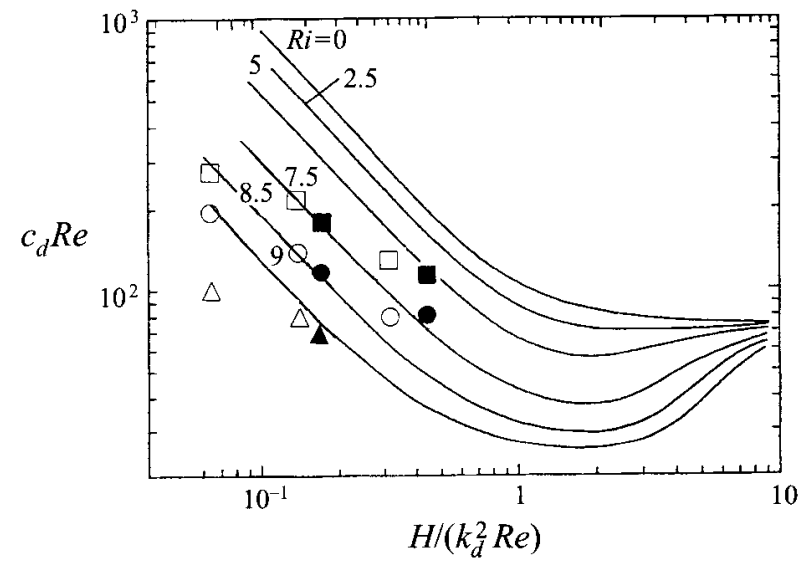

FIGURE 5. Drag coefficient as a function of $H$ for various $R i$. Solid lines are the theoretical results. Open $(R e=200)$ and closed $(R e=150)$ symbols are numerical data at $R i=9(\triangle, \mathbf{\Delta}) ; 7.5(\bigcirc, \bullet)$; $5(\square, \boldsymbol{\square})$.

outside the range of validity of the theory. Nonetheless, whilst neutral flow results would certainly not satisfy the accuracy criterion (perhaps even at $R e=200$ ), we show later that for larger $R i$ there is at least qualitative agreement between the numerical results and the asymptotic behaviour deduced above.

Figure 5 shows the asymptotic results for the drag. The stratification leads to a decrease in drag and it should be emphasized that in our theory this is a purely inviscid effect, because the drag is determined by the value of $C$ (equation (4.14)) which is determined by the Sadovskii flow. Since the velocity of 'heavy' fluid moving upward will decrease (relative to that in neutral flow), velocity gradients in the stratified Sadovskii flow will be lower than in neutral flow so that the velocity and vorticity distributions will be smoothed out. Although the reduction in drag with decreasing blockage $(H)$ is monotonic in neutral flow it is clearly not in cases with significant stratification. For $R i=9$, for example, the drag reaches a minimum around $H /\left(k_{d}^{2} R e\right)=2$ after the initial rapid fall with increasing $H$, as in neutral flow, but for further increases in $H$ the stratification becomes increasingly weak on the eddy scale and 


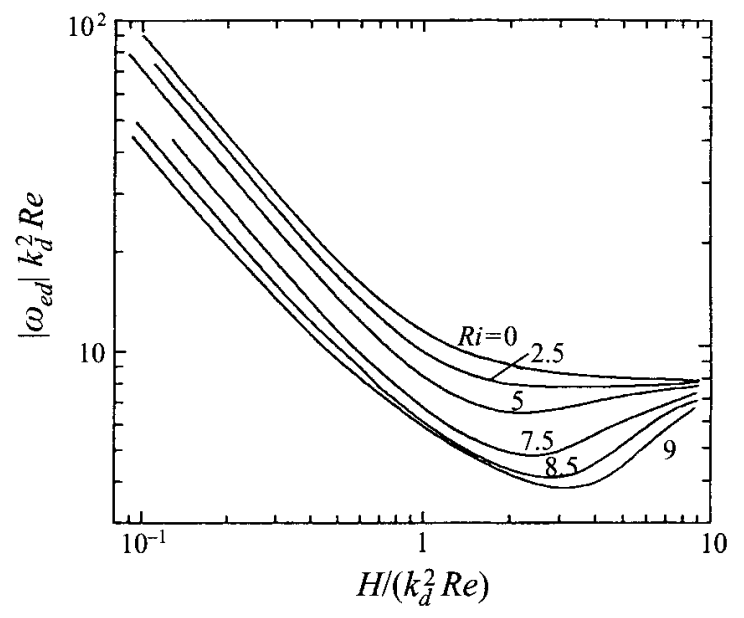

FIGURE 6. Eddy vorticity as a function of $H$ for various $R i$.

the drag rises again towards the neutral flow value. For a wide channel, therefore, the drag reduction with $R i$ is smaller than for narrow channels because of the smaller effective Richardson number on the eddy scale; eventually, of course, the stratification will have no effect at all (i.e. as $\left.H /\left(k_{d}^{2} R e\right) \rightarrow \infty\right)$. Note that for moderate $R e$ the asymptotic theory overestimates the eddy width (not shown here), for reasons which are unclear ( $\mathrm{CH} 2$ ), and this can presumably be expected for stratified flow also. Correspondingly, it is expected that the influence of the stratification will be overestimated. The numerical results included in figure 5 are discussed later.

Figure 6 shows the asymptotic results for the vorticity in the eddy. The nature of the influence of the stratification on the eddy vorticity (and on the eddy length) is different for long $(L \gg H)$ and for short $(L \ll H)$ eddies. In the former case the eddy vorticity is determined by momentum conservation in the effectively inviscid reattachment region, as discussed in $\mathrm{CH} 3$, so that $\omega H=L_{\infty}(R i)$. This is clearly seen in figure 6 where, at small $H /\left(k_{d}^{2} R e\right)$ and for constant Reynolds number, the vorticity is inversely proportional to the channel size with the proportionality constant decreasing with increasing $R i$.

For very short eddies the eddy width is also small in comparison with the channel size. In contrast to the case of long eddies, the influence of both the channel wall and stratification is therefore almost negligible and, as is seen from the numerical calculations of the Sadovskii flow with small $\Gamma$ (the specified circulation around the eddy), the parameters $\alpha$ and $b$-which characterize the eddy shape-are almost independent of $R i$ (see CH3). Similarly, $A W^{2} / \Gamma$ depends only weakly on $R i$ and is close to 0.4 , its value in unbounded Sadovskii flow. However, as $R i$ increases from 0 to 9 , the value of $C$ (for, say, $\Gamma=0.5$ ) decreases by about $20 \%$, from 74 to 60 , and $L$ behaves similarly. This decrease in $C$ (i.e. in drag) results in a decrease in the vorticity flux from the body and this in turn leads to a decrease in the eddy vorticity, as seen for the largest $H / k_{d}^{2} R e$ in figure 6 .

These results have a simple interpretation. For $L \ll H$ the influence of stratification manifests itself largely as a decrease in the effective velocity of the flow past the eddy on the eddy scale (i.e. the velocity at infinity). However, this is not the only effect: stratification also has a direct influence on the eddy flow itself. The vorticity balance in the eddy is affected by an interesting feature arising from the vorticity present in the free stream. Unlike the non-stratified case, vorticity is not conserved and is not zero 


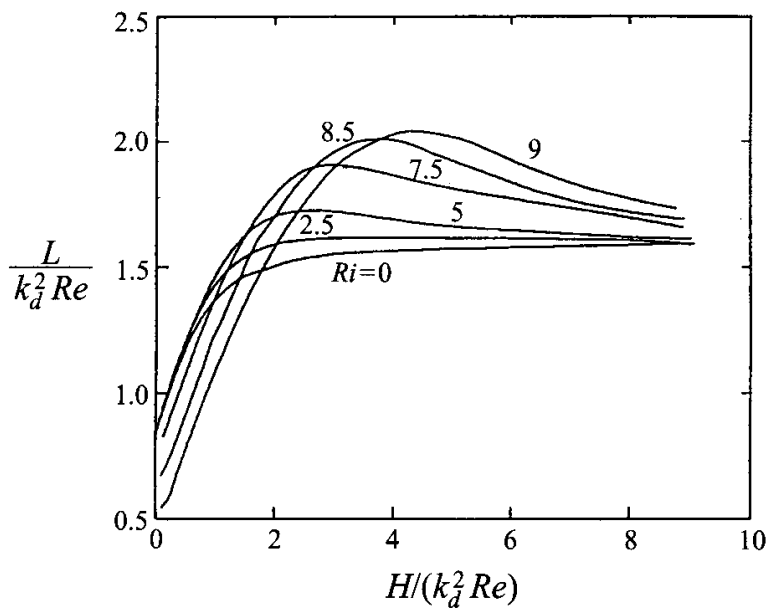

FIGURE 7. Eddy length as a function of $H$ for various $R i$.

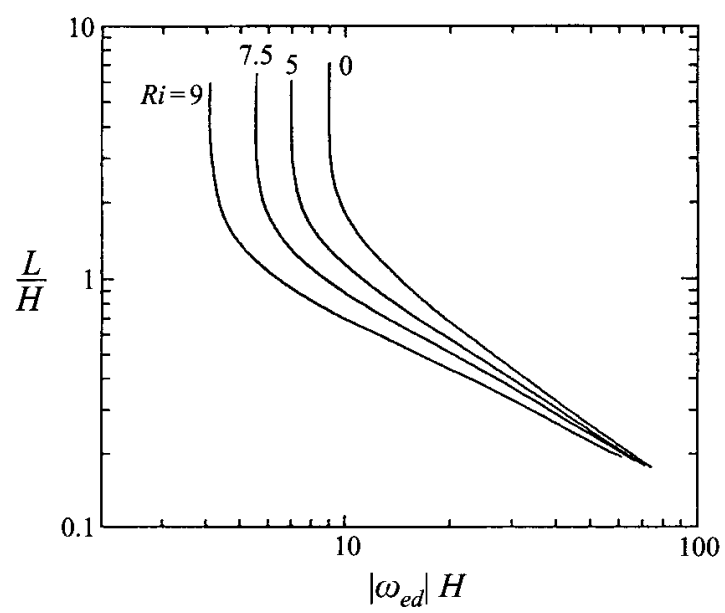

FIGURE 8 . The dependence of eddy length on the eddy vorticity in the Sadovskii flow (see Chernyshenko 1993).

above the eddy. It has the same sign there as it has inside the eddy and it diffuses into the eddy. For a given eddy shape, this stratification-generated vorticity is, interestingly, proportional to the vorticity inside the eddy. Equation (4.16) describes this in detail but it is most easy to understand in terms of dimensional considerations: in stratified Sadovskii flow the non-dimensional vorticity ratio depends only on the choice of the particular member of the family of solutions (see $\mathrm{CH} 3$ ) and therefore the vorticity generation phenomenon enters the vorticity balance condition only as a factor $\left(1+g_{\infty} R i S / 2 H^{2}\right)$ on $\omega_{e d}$. For short eddies $S / H^{2}$ is small and the effect we are discussing is therefore also small.

Because of the influence on the vorticity balance of the stratification-generated vorticity, the eddy length is also affected and its variation with $H$ is shown in figure 7 . In discussing these results it is important to note the dependence between the eddy vorticity and the eddy size, so this is shown in figure 8 . In wide channels (i.e. large $\left.\left|\omega_{e d}\right| H\right)$ it is clear that $L \omega_{e d} \approx$ constant, while in narrow channels $\omega_{e d}$ does not depend on the eddy length and decreases as stratification increases. For short eddies the influence of stratification on the eddy scale is small and so the product $\omega_{e d} L$ is almost 


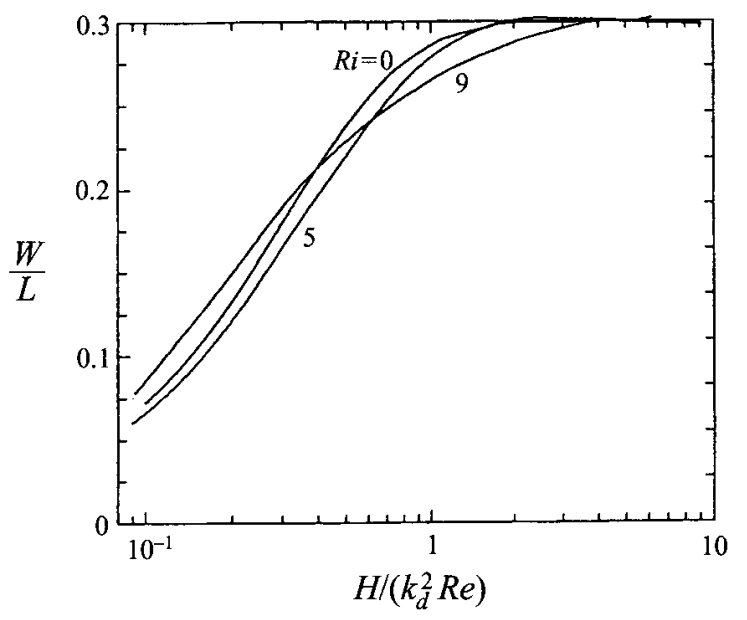

Figure 9. The eddy shape. In a wide channel (e.g. $H / k_{d}^{2} R e=1$, say) the aspect ratio decreases with increasing $R i$ but in a narrow channel the dependence is not monotonic.

constant. Correspondingly, the decrease in the eddy vorticity (explained above) results in an increase in eddy length, as seen in figure 7 (for large $H / k_{d}^{2} R e$ ).

For long eddies the vorticity depends only on $R i$ and tends to zero for $R i \rightarrow \pi^{2}$, as discussed above. In this case the drag is proportional to $L$ and the proportionality constant decreases with $R i$, in agreement with the 'smoothing' of the velocity field discussed earlier; it does not, however, tend to zero for $R i \rightarrow \pi^{2}$. A decrease in eddy vorticity requires a decrease in the vorticity flux from the body. Since part of the eddy vorticity is supplied by diffusion from outside the eddy, this vorticity flux from the body decreases further and the total decrease in flux leads to a substantial decrease in drag (figure 5, small $H / k_{d}^{2} R e$ ) and a corresponding decrease in eddy length (figure 7, small $H / k_{d}^{2} R e$ ).

Finally, figure 9 shows how the eddy shape, measured by the ratio of its width to its length, varies with channel size for various degrees of stratification. For large enough channels stratification has only a weak effect since, as noted earlier, the stratification scale is then much larger than the eddy scale. However, for smaller channels (i.e. $H /\left(k_{d}^{2} R e\right)<0.6$, say) the variation in $W / L$ is not monotonic. Here stratification first acts to reduce the aspect ratio by helping to 'squash' the eddy (much as increasing blockage does) but eventually the reduction in $W$ is more than counteracted by the reduction in $L$ (see figure 7 ) so that $W / L$ increases again.

\subsection{Comparisons with experiment}

We now compare the asymptotic behaviour discussed earlier with results from both numerical and physical experiments. For the former we use previous data from Castro (1993) and some new data obtained using essentially the same, steady Navier-Stokes code. $\uparrow$ The latter will therefore not be described in detail; instead we give a summary of the salient features. Equations (1.1)-(1.3) (with $S c=1000$ ) were discretized on a staggered rectangular non-uniform grid and solved using fairly standard iterative ADI methods, with an additional pressure perturbation equation solved with equation (1.3)

$\dagger$ A referee has enquired about the possibility that steady solutions for $K<1$ may not always exist. Certainly, for $K>1$, periodic unsteadiness occurs in certain parameter ranges (e.g. Castro et al. 1990; Hanazaki 1989), so that the time-dependent version of equations (1.1)-(1.3) are required, but we know of no clear evidence for the existence of unsteadiness at $K<1$, nor any physical reasons for such unsteadiness other than those which determine transition to turbulence. 
using SIMPLER (Patankar 1980), which is a more efficient version of the well-known SIMPLE algorithm. It was particularly important to ensure accurate differencing of the convective terms. Results in Castro (1993) were obtained using a second-order upwinding (HUD) scheme on all equations or HUD on the density equation and a third-order scheme (QUICK) on the momentum equations. Neither of these are strictly bounded schemes and more recent computations have used a steady flow version of the bounded, higher-order scheme first developed for unsteady compressible flows (Van Leer 1974).

Care was taken to ensure adequate grid resolution. Grids having at least $100 \times 45$ nodes in the $(x, y)$ directions, respectively, were used but many runs had much larger mesh sizes (up to $300 \times 170$ ). Grid expansion and contraction ratios were kept below 1.25 , which has been shown to be adequate for these kinds of flows provided higherorder schemes are used (Castro \& Jones 1987). A simple fence (of unit height) was used as the body and was placed typically 50 heights from the domain inlet. Mesh sizes at the fence tip were below about 0.02 to ensure adequate resolution in the region where vorticity is shed from the body. Note that since primitive variables are used, with neither pressure nor velocity mesh points coinciding with the corner, there is no 'corner singularity difficulty' of the kind encountered if a vorticity/stream function formulation is employed, although fine grids are especially important in the corner region and particular care is necessary in formulating the finite difference expressions (Castro, Cliffe \& Norgett 1982). Many of the runs also had relatively fine meshes ( $\Delta x$ about 0.1 ) around the end of the recirculating region. The boundary conditions simulated those used in the asymptotic theory, except that zero-gradient conditions were imposed at the outlet. Some tests were included to ensure that the results did not depend significantly on the location of this boundary, which was always at least $2 L$ downstream of the body location.

We do not claim that our results are all entirely free from numerical error, but a number of grid-dependancy tests suggested that such errors are not significant. As a further check on accuracy we compared some salient $R i=0$ results with those previously obtained by Natarajan, Fornberg \& Acrivos (1993), who used a much more sophisticated and well-tested numerical scheme. In the case of our earlier results for $R e=50, L$ and $C_{d}$ were 28.62 and 1.452 , respectively, at $H=20$, with corresponding values of 17.06 and 2.625 at $H=5$. Natarajan et al. give 29.13, 1.46 at $H=20$ and $20.88,2.55$ at $H=5$. The differences between the two sets of results are below $2 \%$ at $H=20$, but much larger at $H=5$; this is undoubtedly due to the different boundary condition employed at $y=H$. As noted earlier, Castro (1993) forced $U=1$ there (simulating a towing tank condition) whereas Natarajan et al. imposed symmetry. Results for $H=10$ from the present computations (which employed a symmetry condition at $y=H$ ) have also been compared with those of Natarajan et al., in the range $50<R e<250$. The differences in $L$ and $C_{d}$ are in all cases less than $1 \%$ and usually much lower, which gives confidence that the present calculations are sufficiently accurate for our purpose here.

In order to test the asymptotic theory for large values of the parameter $H / k_{d}^{2} R e$ it is ideally necessary to use much larger values of $H$ than we could reach in the present computations. For $R e=200, R i=0$ we found it impossible to obtain properly converged solutions for $H$ larger than about 10 . This difficulty was increasingly less acute with increasing stratification; for $R i=7.5$, for example, it was possible to obtain solutions for $H$ values up to at least 50. Smaller $R e$ allowed us to move to higher $H$ and, in general, we found that for a given $R e$ reliable computations (with our numerical scheme) were only possible for channel widths up to around the 'critical' value for 


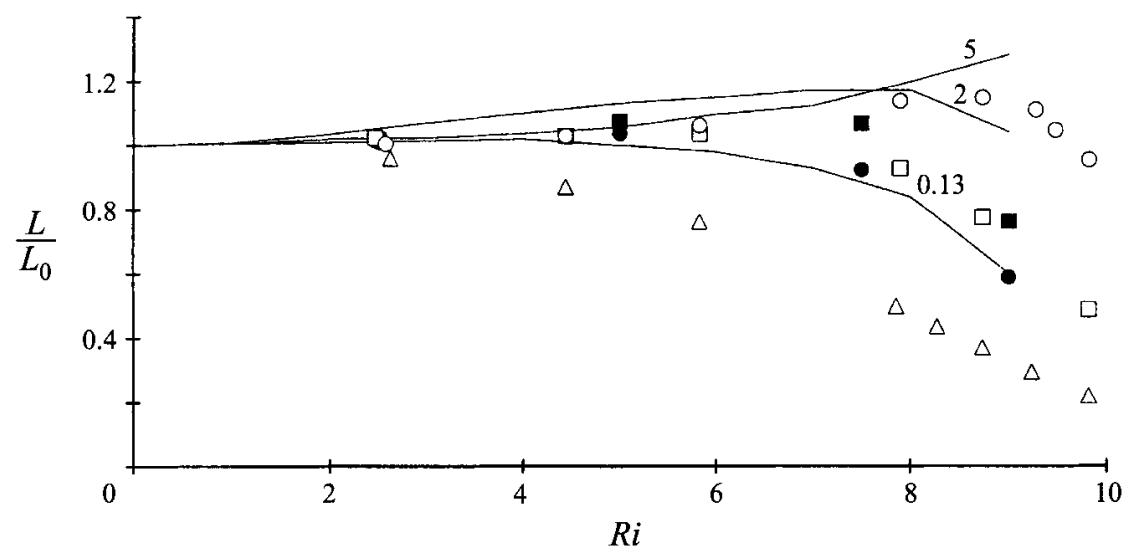

FIgURE 10. Eddy length as a function of $R i$ for various $H / k_{d}^{2} R e$. Note that $L$ is normalized by its value for $R i=0$. Symbols as for figure 11 .

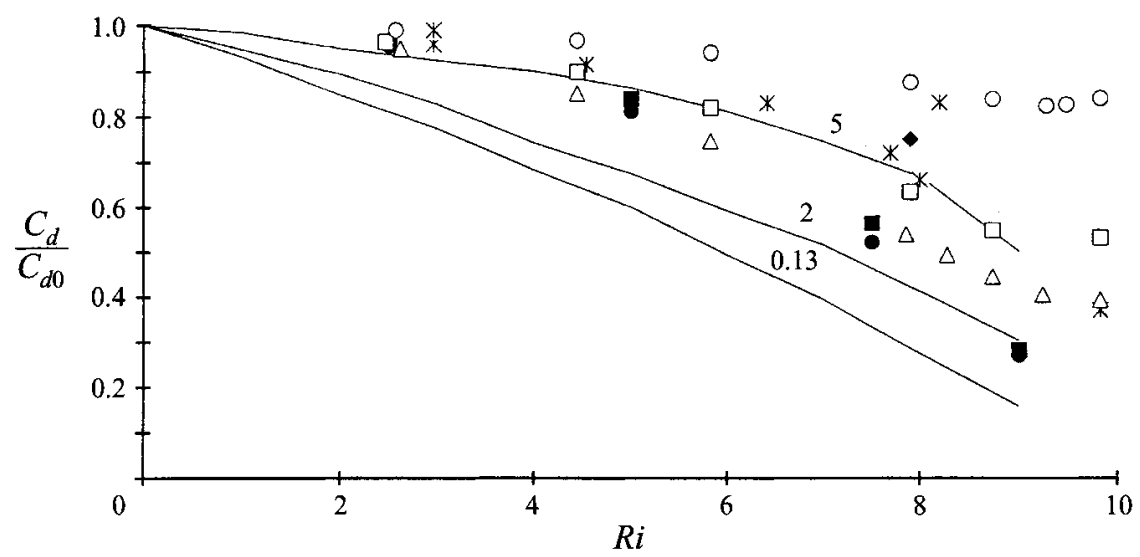

FIGURE 11. Drag coefficient as a function of $R i$ for various $H / k_{d}^{2} R e$. Note that $c_{d}$ is normalized by its value for $R i=0$. Solid lines are theoretical results at the values of $H / k_{d}^{2} R e$ given. ${ }^{*}$ are from physical experiments. Other symbols are: $\triangle, R e, H / k_{d}^{2} R e=50,0.129 ; \square, 50,0.517 ; O, 50,1.29$; $\square, 150,0.172 ; 0,200,0.065$ (present results); $\bullet$, 20, 0.98 (from Hanazaki 1989).

which the transition to the wide-wake solution began (see Natarajan et al. 1993 and $\mathrm{CH} 2$ ). This could be partly a result of the greater difficulty in constructing suitable meshes for such flows, but may also be an indication that in this parameter range the solutions are unstable, so require quite different numerical approaches (Fornberg 1991 and Natarajan et al. used a method which will yield valid solutions whether or not they are stable).

The data obtained from physical experiments are largely those published previously (Castro \& Snyder 1990 and Castro et al. (1990). These were from experiments in a large towing tank, with salt used as the stratifying medium and fences of various heights submerged below the free surface, which acted essentially as a symmetry plane. The bottom boundary condition (zero wall velocity) was, as noted earlier, not quite that used in the theory or the numerical computations, but the effects of this difference are likely to be small for the cases shown here.

The most useful way of making comparisons with the theory is to study the changes in drag and eddy length that arise at constant $R e$ and $H$ as the stratification is varied. This is done in figures 10 and 11 which show $L$ and $c_{d}$, normalized by their values in 
neutral flow ( $L_{0}$ and $c_{d 0}$, respectively) and plotted as functions of $R i$ for various values of $H / k_{d}^{2} R e$. Recall that the analytic results described earlier showed that at fixed $R e$ stratification reduces the drag monotonically for all $H$ (figure 11), whereas the eddy length first rises at large $H$. If $H$ is large enough it continues to rise with $R i$ but otherwise it reaches a maximum and subsequently falls. For small channels $L$ falls monotonically. This behaviour is reflected qualitatively in the numerical results although the largest $H$ used in the calculations (50) is clearly not sufficiently large to yield a monotonically rising $L / L_{0}$ for all $R i$. In fact, taking $k_{d}=0.88$ (appropriate to the unbounded Kirchhoff flow over a flat plate) $H / k_{d}^{2} R e$ is about 1.3 for the $R e=50$ case, which is within the range predicted to give a non-monotonic variation in $L$ (see also figure 7). However, $R e=50$ is smaller than required for the asymptotics to be really adequate (at least at $R i=0$ ). This may be partly why the drag reductions are much smaller than suggested by the theory, although recall that we expect the latter to overestimate the effect of stratification on drag $(\$ 5.1)$. We include in figure 11 the result from the only other case in the literature of a comparable computation (of which we are aware). This is Hanazaki's (1989) calculation for $H=15, R e=20, K=0.8$ (so that $R i=7.9$ and $H / k_{d}^{2} R e=0.97$ ). Although, again, $R e$ is lower than strictly required by our theory, the result for $c_{d}$ and falls nicely within the trend (near $R i=8$ ) indicated by the present results and our theory.

The higher- $R e$ data, corresponding to $H / k_{d}^{2} R e=0.065$ and 0.172 , are likely to be a rather better test of the theory. Figure 10 shows that the behaviour of the eddy length with increasing stratification is, again, in qualitative agreement with the theory. In these cases the drag falls much more significantly with $R i$, as predicted by the theory, although the fall is not so rapid as the latter would suggest. Note that the most rapid fall in $L$ shown by the computations (figure 10) is for the case $R e=50, H=5$. These values yield $H / k_{d}^{2} R e=0.129$, which is relatively close to the value $(0.172)$ for the $R e=150, H=20$ case. Although the drag variation is similar (figure 11), the latter case has an eddy length variation more in line with the theory, as expected because the Reynolds number is significantly larger. (Recall also that the $R e=50, H=5$ computations of Castro 1993 used a different $y=H$ boundary condition than that assumed in the theory and used in the present computations.) The $R e=150$ and 200 results for $c_{d}$ at $R i=5,7.5$ and 9 are included in figure 5 . It is clear that agreement with the theory improves for increasing $H / k_{d}^{2} R e$ and, at a fixed value of the latter, is better at larger $R i$, as anticipated from the accuracy considerations discussed earlier.

Figure 11 includes the drag data obtained from the physical experiments. All the experiments were at high Reynolds number (about 20000), so that the wake was fully turbulent. The eddy length was not measured, but by comparison with wind tunnel experiments on flow over flat plates with central splitter plates, might be expected to be around 20 (at $R i=0$ ). This is consistent with the visual observations made during the towing tank experiments, which typically had $H=20$. Our computations (and those of Natarajan et al. 1993) suggest that for a fully laminar case $R e=33$ would yield $L=20$ for $H=20$ so, assuming that the variation of eddy viscosity in the turbulent wake is such as to make the effective Reynolds number about 30 , we deduce an effective value of about 0.9 for $H / k_{d}^{2} R e$. This is, of course, far from rigorous, but the experimental data on figure 11 are, on that basis, quite consistent with the numerical results - showing a somewhat lower fall with $R i$ than occurs for, say, $H / k_{d}^{2} R e=0.172$, as expected on the basis of the theory.

We emphasize here that this 'effective Reynolds number' argument, although not rigorous, must be qualitatively correct. At Reynolds numbers high enough to give a fully turbulent wake, the separated region is very much shorter than it would be if 

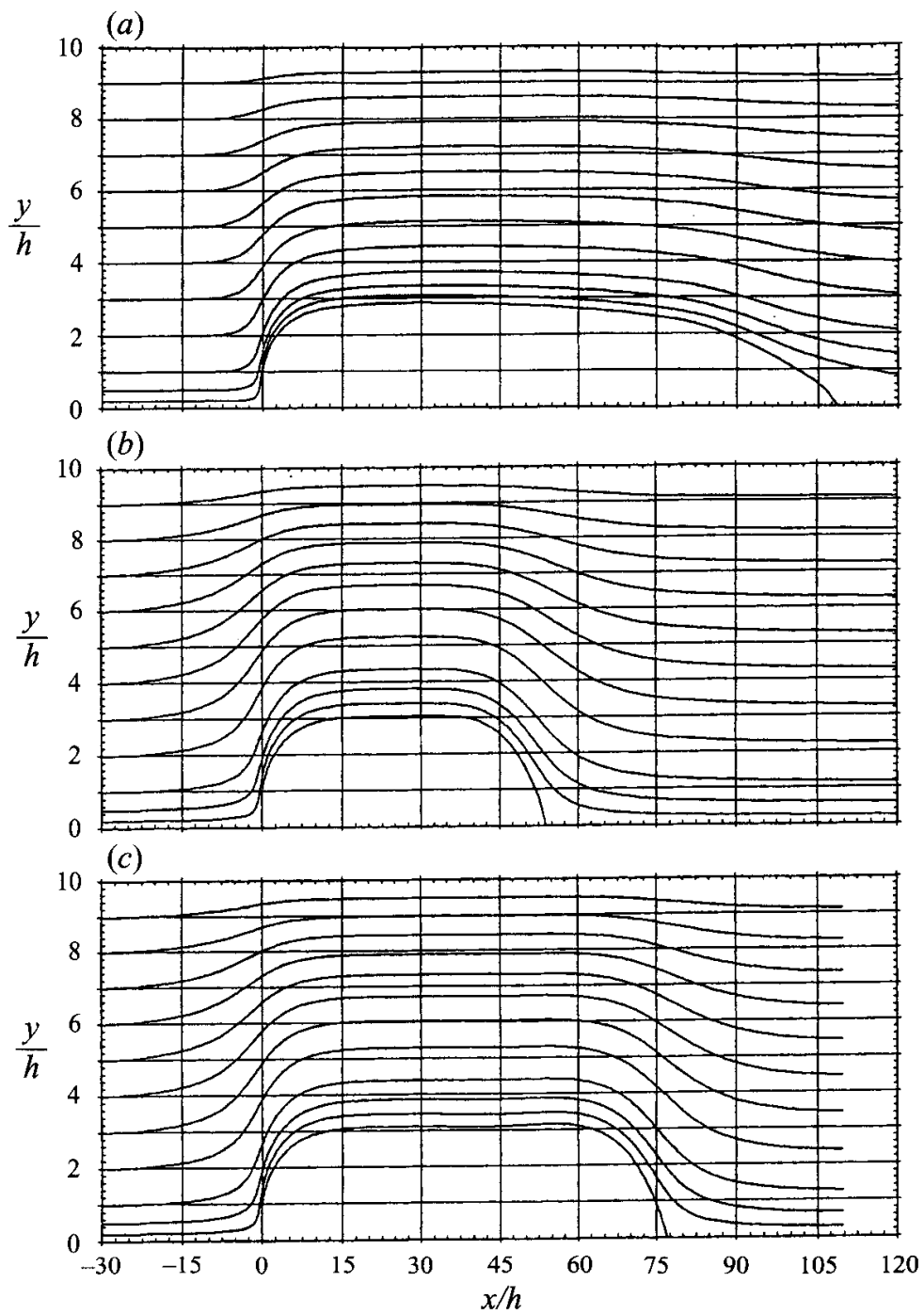

FiguRe 12. Streamlines for $H=10$. (a) $R e=210, R i=0$; (b) $R e=200, R i=8.9$; (c) $R e=300, R i=8.9$.

laminar conditions could be maintained. Indeed, neither its length nor the 'effective' Reynolds number is any longer a function of the physical Reynolds number, providing the latter is high enough. The laminar flow surrounding the body and its lee-side (turbulent) recirculation region and wake will then be qualitatively similar to that around the same body but at an 'effective' Reynolds number low enough to give a laminar eddy of the same length. This flow will correspond to the theoretical one prior to the wide-wake transition; recall that we could not obtain converged numerical solutions in the wide-wake regime, deducing that such flows are probably unstable they have certainly never been identified in a physical experiment.

One further feature of our numerical results is worth noting. Figure 12 shows streamlines for the $H=10$ case with $R i=0$ and 8.9 . The flow for $R i=8.9$ is very similar in form to the asymptotic 'long-eddy' Sadovskii regime identified in $\mathrm{CH} 3$; the wake is roughly symmetric front-to-back and in the central region it is quite flat, simply stretching as $R e$ increases (compare figures $12 b$ and $12 c$ ). In these two cases the 
reattachment process is essentially inviscid, whereas for $R i=0$ inviscid reattachment at these Reynolds numbers is not possible. It is interesting that the wake width, $W$, is about 3 for the long-eddy solutions, compared with the corresponding asymptotic result (identified in $\mathrm{CH} 3$ ) of about 4.6 for the case in which no body is present. Turfus' (1993) work implies that the long-eddy (no body) $\mathrm{CH} 3$ result of $W / H=2 / 3$ for $R i=0$ reduces to about 0.57 for $H=10$, so a thinner eddy, compared with the $\mathrm{CH} 3$ results, might on that basis be expected in stratified cases also. However, a more likely explanation for the difference is simply that $R e$ and/or $H$ are not sufficiently large in the computations for very close agreement with the asymptotics to be expected. Some evidence for this is provided by calculations we made of the inviscid flow without the body for an eddy having the vorticity which emerges from our Navier-Stokes computations (with the body). These gave eddy widths very close to the latter. In any case, there is no doubt about the eddy structure identified in our computations and we believe that direct numerical evidence for the very-long-eddy structure identified in $\mathrm{CH} 3$ has not previously been reported.

As a final comment, it might well be instructive to obtain numerical solutions at larger values of $H / k_{d}^{2} R e$ than could be achieved here (by using more appropriate numerical methods). However, the present results, taken together, certainly have the qualitative features expected on the basis of our theory and thus provide some confirmation of the latter.

\section{Conclusions}

An extension of the theory of Chernyshenko \& Castro (1993) for the asymptotic flow at high Reynolds number over a bluff body in a channel has been described for the corresponding case of flows with stable density stratification. The theory is valid for $R e \gg 1, H \gg 1, R i<\pi^{2}$, and it is shown that the influence of the stratification $(R i)$ on the separated wake depends critically on the channel width (strictly, on $H /\left(k_{d}^{2} R e\right)$ ). For large channels, the stratification scale is large compared with the eddy scale so that the effects are not large. In this regime, stratification acts to reduce the drag, $c_{d}$, and the eddy vorticity, $\omega_{e d}$, by about $20 \%$ at $R i=9$, with a corresponding increase in the eddy length, $L$. For narrow channels the behaviour of $c_{d}$ and $\omega_{e d}$ is in the same sense, but the changes are very much larger and $L$ is reduced significantly. Here, the additional vorticity created in the external flow by the stratification, which diffuses into the eddy, has a much more significant effect on the overall flow. Changes in $L$ at fixed $R e$ and $H$ can be non-monotonic at intermediate channel widths. For all $H$ and at sufficiently large $R e$ the flow approaches the stratified Sadovskii flow discussed by Chernyshenko (1993).

Many of the effects, notably the reduction in drag with stratification, are essentially inviscid mechanisms. They are therefore expected to occur also in high-Reynoldsnumber turbulent flows. The limited data available in the literature substantiate this conclusion. In that case the 'effective' Reynolds number (the value indicated by numerical computations to yield a laminar eddy of similar length to the fully turbulent one) is, in fact, much lower but still high enough for the asymptotic theory to give the correct qualitative trends. Our (laminar) numerical computations at moderate Re have also been shown to demonstrate the same behaviour as that predicted by the asymptotic theory, including the non-monotonic variation of eddy length with increasing stratification (although more detailed quantitative comparisons with numerical data for the wide channel regime must await more sophisticated computations). We therefore conclude that the physical mechanisms revealed by the 
theory apply to realistic flows. Our results suggest, further, that (under the restrictions mentioned in §1) the asymptotic theory given by Chernyshenko (1988) and Chernyshenko \& Castro (1993) provides a useful tool for revealing physical mechanisms in real flows, especially in cases when such flows are distorted by additional effects like, in the present case, density stratification. We suggest that the effects of other complications, like compressibility, rotation or three-dimensionality, could be studied in a similar framework.

The work was started while one of us (S. I.C.) was visiting the University of Surrey, with financial support from the Royal Society, which is therefore gratefully acknowledged, as is later support provided by the Russian Foundation for Fundamental Research under project no. 93-103-17622. We also benefited from some helpful comments from the Referees.

\section{Appendix. The calculation of $g_{\infty}$ for a given Sadovskii flow}

The value of $g_{\infty}$ is to be found from (4.12), rewritten here for convenience:

Here

$$
\begin{aligned}
& \partial g / \partial t=\partial^{2} g / \partial z^{2}+f(t), \quad f(t)=\left\{\begin{array}{l}
-1, z>0 \\
+0, z<0,
\end{array}\right. \\
& t=0, \quad z>0, \quad g=0 \\
& z<0, \quad g(0, z)=g(1, z), \\
& z \rightarrow-\infty, \quad|g|<\infty, \\
& z=0, \quad b<t<1, \quad g=0 \\
& f(t)=\sin \Theta / U
\end{aligned}
$$

For the given Sadovskii flow $f(t)$ and $b$ are known. Then (A 1) determines $g_{\infty}$ as a functional of $f(t)$ and a function of $b$. Let $r(z)=g(0, z), z<0$. Then by the well-known formula for the solution of a heat equation

$$
\begin{aligned}
g(t, z)=\frac{1}{(4 \pi t)^{1 / 2}} \int_{-\infty}^{0} r(\xi) \exp \left[\frac{-(z-\xi)^{2}}{4 t}\right] \mathrm{d} \xi & \\
& -\int_{0}^{t} \int_{0}^{\infty} f(\tau) \frac{\exp \left[-(z-\xi)^{2} /(4(\tau-t))\right]}{2(\pi(t-\tau))^{1 / 2}} \mathrm{~d} \xi \mathrm{d} \tau, \quad t<t \leqslant b .
\end{aligned}
$$

Denoting $\int_{0}^{\infty} \mathrm{e}^{-x^{2}} \mathrm{~d} x=\operatorname{erfc}(y)$ and $q(z)=g(b, z)$ we obtain

$$
q(z)=\frac{1}{(4 \pi b)^{1 / 2}} \int_{-\infty}^{0} r(\xi) \exp \left[\frac{-(z-\xi)^{2}}{4 b}\right] \mathrm{d} \xi-\frac{1}{\pi^{1 / 2}} \int_{0}^{b} f(\tau) \operatorname{erfc}\left[\frac{-z}{2(b-\tau)^{1 / 2}}\right] \mathrm{d} \tau .
$$

By a similar well-known formula

$$
r(z)=\frac{1}{[4 \pi(1-b)]^{1 / 2}} \int_{-\infty}^{0} q(\xi)\left[\exp \left(\frac{-(z-\xi)^{2}}{4(1-b)}\right)-\exp \left(\frac{-(z+\xi)^{2}}{4(1-b)}\right)\right] \mathrm{d} \xi .
$$

The solution of this system of integral equations yields $g_{\infty}=\lim _{z \rightarrow-\infty} r(z)$. We introduce the Green function $R(z, \tau)$ so that, for all $\mathrm{f}(\tau)$,

$$
r(x)=\int_{0}^{b} R(z, \tau) f(\tau) \mathrm{d} \tau
$$


Substituting $f(\tau)=d\left(\tau-\tau^{\prime}\right)$, as usual, into (A 2) and (A 3) leads to the following system of integral equations:

$$
\begin{aligned}
& Q(z, \tau)=\frac{1}{(4 \pi b)^{1 / 2}} \int_{-\infty}^{0} R(\xi, \tau) \exp \left[\frac{-(z-\xi)^{2}}{4 b}\right] \mathrm{d} \xi-\frac{1}{\pi^{1 / 2}} \operatorname{erfc}\left(\frac{-z}{2(b-\tau)^{1 / 2}}\right), \\
& R(z, \tau)=\frac{1}{[4 \pi(1-b)]^{1 / 2}} \int_{-\infty}^{0} Q(\xi, \tau) \exp \left[\left(\frac{-(z-\xi)^{2}}{4(1-b)}\right)-\exp \left(\frac{-(z+\xi)^{2}}{4(1-b)}\right)\right] \mathrm{d} \xi .
\end{aligned}
$$

Then

$$
g_{\infty}=\int_{0}^{b} R(-\infty, \tau) f(\tau) \mathrm{d} \tau .
$$

System (A 4) and (A 5) was solved numerically. For these calculations the substitutions $Q(z, \tau)=V(y, \tau / b), z=2 y b^{1 / 2}$ and $R(z, \tau)=W(y, \tau / b)$ were used. Denoting $\gamma=$ $[b(1-b)]^{1 / 2}$ we obtain

$$
\begin{aligned}
& V(y)=\frac{1}{\pi^{1 / 2}} \int_{-\infty}^{0} W(\xi) \exp \left[-(y-\xi)^{2}\right] \mathrm{d} \xi-\frac{1}{\pi^{1 / 2}} \operatorname{erfc}\left[-y /(1-\tau / b)^{1 / 2}\right], \\
& W(y)=\frac{\gamma}{\pi^{1 / 2}} \int_{-\infty}^{0} V(\xi) \exp \left[-\gamma^{2}(y-\xi)^{2}\right]-\exp \left[-\gamma^{2}(y+\xi)^{2}\right] \mathrm{d} \xi .
\end{aligned}
$$

Functions $V(y)$ and $W(y)$ were represented by their values on a uniform grid for $y_{m} \leqslant y \leqslant 0$ and by constant values $V_{\infty}$ and $W_{\infty}$ for $y<y_{m}$. Then the integrals from $-\infty$ to $y_{m}$ can be calculated explicitly. This gives

$$
\begin{gathered}
V\left(y_{i}\right)=\frac{1}{\pi^{1 / 2}} \int_{x_{m}}^{0} W(\xi) \exp \left[-\left(y_{i}-\xi\right)^{2}\right] \mathrm{d} \xi+\frac{W_{\infty}}{\pi^{1 / 2}} \operatorname{erfc}\left(y_{i}-y_{m}\right) \\
-\frac{1}{\pi^{1 / 2}} \operatorname{erfc}\left[-y_{i} /(1-\tau / b)^{1 / 2}\right], \\
\begin{array}{c}
W\left(y_{i}\right)=\frac{\gamma}{\pi^{1 / 2}} \int_{x_{m}}^{0} V(\xi)\left\{\exp \left[-\gamma^{2}\left(y_{i}-\xi\right)^{2}\right]-\exp \left(-\gamma^{2}\left(y_{i}+\xi\right)^{2}\right)\right\} \mathrm{d} \xi \\
+\frac{V_{\infty}}{\pi^{1 / 2}}\left[\operatorname{erfc}\left(\gamma\left(y_{i}-y_{m}\right)\right)-\operatorname{erfc}\left(\gamma\left(y_{i}+y_{m}\right)\right)\right] \\
y_{i}=y_{m}(i-1) /(m-1), \quad y_{m}<0, \quad i=1, \ldots, m .
\end{array}
\end{gathered}
$$

This system was solved iteratively. The integrals on the right-hand side were calculated by a forth-order Simpson method. Results are presented in figure 13. As $b$ for the Sadovskii flows is always in the range $0.5 \leqslant b \leqslant 0.55$ (see Chernyshenko 1993) a linear approximation is appropriate to find $R(-\infty, \tau)$ for other $b$. As seen in the figure, $R(-\infty, \tau)$ can be approximated with reasonable accuracy by the linear function of $\tau / b$ :

$$
R \approx k(b)(\tau / b-1) .
$$

This allows considerable simplification in the further computations. For $k(b)$ the linear interpolation formula, $k(b)=0.2797+0.7(b-0.5)$, was used. Substituting (A 7) and $f(\tau)$ from (A 1) into (A 5) yields

$$
g_{\infty}=\int_{0}^{b} k(b)(t / b-1) \sin \theta / U \mathrm{~d} t .
$$




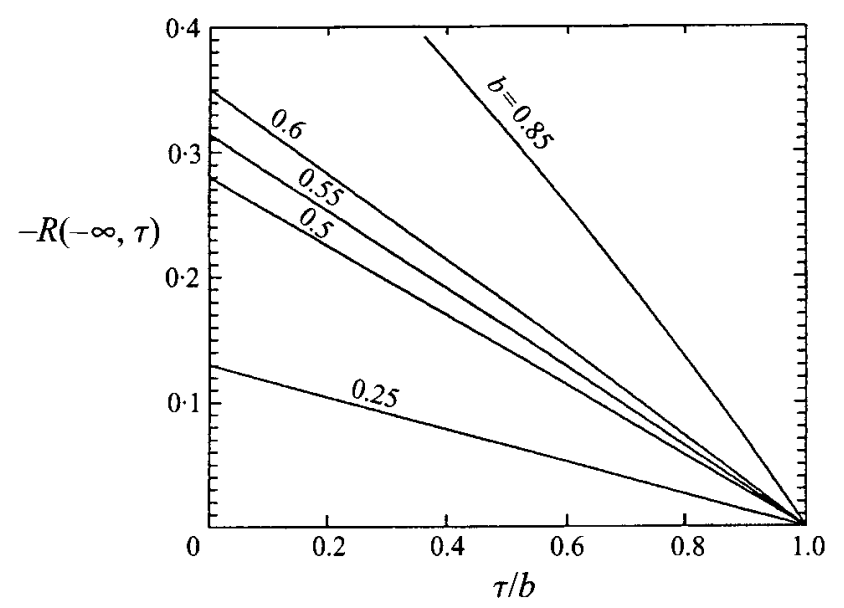

FIGURE 13. Solutions for the Green's function (A 5) for various $b$. Note that the lines are not exactly straight.

Since $\sin (\theta)=0$ at $t=b / 2$, a small deviation of $R$ from the linear approximation used here leads to a very small error. Recalling the definitions $\mathrm{d} t=\mathrm{d} s / U, \sin \theta \mathrm{d} s=\mathrm{d} y$ and noting that $\left.\sin \theta\right|_{s}=-\left.\sin \theta\right|_{s_{B}^{-s}}$ (A 8) yields

$$
\begin{aligned}
g_{\infty}=k \int_{0}^{s_{B}}\left(\frac{t}{b}-1\right) \frac{\sin \theta}{\Gamma} \mathrm{d} s=\frac{k}{\Gamma b} \int_{0}^{s_{B}} t \sin \theta \mathrm{d} s=\frac{k}{\Gamma b} \int_{s=0}^{s=s_{B}} t \mathrm{~d} y \\
=-\frac{k}{\Gamma^{2} b} \int_{s=0}^{s=s_{B}} y U \mathrm{~d} s=-\frac{k}{\Gamma^{2} b} \int_{s=0}^{s=s_{A}} y U \mathrm{~d} s .
\end{aligned}
$$

The last integral is the circulation of the vector $y u$ around the upper half of the eddy in the Sadovskii flow, with the sign reversed due to the clockwise direction of integration. Here $u=(u, v)$ is the velocity vector in the Sadovskii flow and $y$ is the vertical coordinate. Using Stokes' theorem we obtain

$$
g_{\infty}=\frac{k}{\Gamma^{2} b} \iint_{\text {eddy }} \operatorname{rot}(y u, y v) \mathrm{d} x \mathrm{~d} y=\frac{k}{\Gamma^{2} b} \iint_{\text {eddy }}(y \omega-u) \mathrm{d} x \mathrm{~d} y=\frac{k \omega_{e d}}{\Gamma^{2} b} \iint_{\text {eddy }} y d x \mathrm{~d} y .
$$

The integral here is taken over the upper half of the eddy in the Sadovskii flow. The same program as that used by Chernyshenko (1993) was used to compute the last integral for different $R i$ and $H / L$.

Quantitative results obtained in this Appendix were checked in two ways. First, the behaviour of the Green function for $b \ll 1$ was derived and found to be consistent with the numerical results. Secondly, for $f(t)=\delta(t)$ the problem (A 1$)$ is a special case of the problem considered in Chernyshenko (1982). Agreement with the latter was quite satisfactory.

\section{REFERENCES}

Boyer, D. L., Davies, P. A., Fernando, H. J. S. \& Zhang, X. 1989 Linearly stratified flow past a horizontal circular cylinder. Phil. Trans. R. Soc. Lond. A 328, 501-528.

CASTRO, I. P. 1993 Effects of stratification on separated wakes: Part I, weak static stability. In Waves and Turbulence in Stably Stratified Flows (ed. S. D. Mobbs \& J. C. King), pp. 323-346. Oxford University Press.

Castro, I. P., Cliffe, K. A. \& Norgett, M. J. 1982 Numerical predictions of the laminar flow over a normal flat plate. Intl J. Numer. Meth. Fluids 2, 61-88. 
Castro, I. P. \& Jones, J. M. 1987 Studies in numerical computations of recirculating flows. Intl J. Numer. Meth. Fluids 7, 793-823.

CASTro, I. P. \& SNYDER, W. S. 1990 Obstacle drag and upstream motions in stratified flow. In Stratified Flows (ed. E. J. List \& G. H. Jirka), pp. 84-98. ASCE.

Castro, I. P., Snyder, W. S. \& Baines, P. E. 1990 Obstacle drag in stratified flow. Proc. R. Soc. Lond. A 429, 119-140.

Chernyshenko, S. I. 1982 An approximate method of determining the vorticity in the separation region as the viscosity tends to zero. Fluid Dyn. 17, no. 1, 1-11 (translated from Izv. Akad. Nauk. SSSR, Mekh. Zhidk. Gaza 1, 10-15).

Chernyshenko, S. I. 1988 The asymptotic form of the stationary separated circumference of a body at high Reynolds number. Appl. Math. Mech. 52, 746 (translated from Prikl. Matem. Mekh. 52(6), 958-966 (referred to herein as CH1)).

Chernyshenko, S. I. 1993 Stratified Sadovskii flow in a channel. J. Fluid Mech. 250, 423-431 (referred to herein as $\mathrm{CH} 3$ ).

Chernyshenko, S. I. \& Castro, I. P. 1993 High Reynolds number asymptotics of the steady flow through a row of bluff bodies. J. Fluid Mech. 257, 421-449 (referred to herein as CH2).

Chomaz, J. M., Bonneton, P. \& Hopfinger, E. J. 1993 The structure of the near wake of a sphere moving horizontally in a stratified fluid. J. Fluid Mech. 254, 1-21.

Courant, R. 1962 Partial Differential Equations. Interscience.

DAVIS, R. E. 1969 The two-dimensional flow of a stratified fluid over an obstacle. J. Fluid Mech. 36, $127-143$.

FORNBERG, B. 1991 Steady incompressible flow past a row of circular cylinders. J. Fluid Mech. 225, 655-671.

HANAZAKI, H. 1988 A numerical study of three-dimensional stratified flow past a sphere. $J$. Fluid Mech. 192, 393-419.

HANAZAKI, H. 1989 Upstream advancing columnar disturbances in two-dimensional stratified flow of finite depth. Phys. Fluids A 1, 1976-1987.

Kamachi, M., Saitou, T. \& Honi, H. 1985 Characteristics of stationary closed streamlines in stratified fluids. Part 1, theory of laminar flows. Tech. Rep. Yamaguchi Univ. 3(4), 295-303.

Lin, J.-T. \& PaO, Y.-H. 1979 Wakes in stratified fluids. Ann. Rev. Fluid Mech. 11, 317-338.

LOFQUist, K. E. B. \& PURTelL, L. P. 1984 Drag on a sphere moving horizontally through a stratified liquid. J. Fluid Mech. 148, 271-284.

Natarajan, R., Fornberg, B. \& ACrivos, A. 1993 Flow past a row of flat plates at large Reynolds numbers. Proc. R. Soc. Lond. A 441, 211-235.

PatanKar, S. V. 1980 Numerical Heat Transfer and Fluid Flow. McGraw-Hill.

SERrin, J. 1959 Mathematical Principles of Classical Fluid Mechanics. Springer.

Turfus, C. 1993 Prandtl-Batchelor flow past a flat plate at normal incidence in a channel - inviscid analysis. J. Fluid Mech. 249, 59-72.

TURner, J. S. 1973 Buoyancy Effects in Fluids. Cambridge University Press.

VAN LeER, B. 1974 Towards the ultimate conservative difference scheme. II Monotonicity and conservation combined in a second-order scheme. J. Comput. Phys. 14, 361-370.

YIH, C.-S. 1980 Stratified Flows. Academic. 